\title{
REPRESENTABILITY OF PARTIAL RECURSIVE FUNCTIONS IN FORMAL THEORIES
}

\author{
WILLIAM E. RITTER
}

1. Introduction. Ehrenfeucht and Feferman have shown [1] that all recursively enumerable sets $X$ of natural numbers are "representable" in any consistent recursively enumerable theory $S$ in which all recursive functions are definable (in the sense of Tarski-MostowskiRobinson [4]) and which has a formula $x_{1} \leqq x_{2}$ satisfying conditions (i), (ii) below for each natural number $n$ :

(i) $\vdash{ }_{s} x_{1} \leqq \bar{n} \equiv x_{1}=\overline{0} \bigvee x_{1}=\overline{1} \vee \cdots \vee x_{1}=\bar{n}$,

(ii) $\vdash{ }_{s} x_{1} \leqq \bar{n} \bigvee \bar{n} \leqq x_{1}$.

(Here $\bar{n}$ is the (closed) numerical term of $S$ corresponding to $n$, i.e. $\Delta_{n}$ of $[4$, p. 44].) (By a construction of Cobham (see [3, p. 121] for details), (ii) is redundant in the presence of (i) and the definability in $S$ of the successor function.) That is, for such an $X$, there is a formula $\Phi\left(x_{1}\right)$ of $S$ (with exactly one free variable $x_{1}$ ) such that for every $n$,

$$
n \in X \Leftrightarrow \vdash_{s} \Phi(\bar{n}) .
$$

The argument is to show that there is some creative set $C$ representable in $S$, from which the result follows by the reducibility of $X$ to $C$ by some recursive function (Myhill). Shepherdson has obtained the result [3] more directly by an elegant adaptation of Rosser-type arguments, much as Bernays obtained results of Myhill on theories. In [2] Ritchie and Young show that in every consistent recursively enumerable extension $S$ of R. M. Robinson's system $R$ ([4, pp. 52-53]), all partial recursive functions $\phi$ are "strongly representable." That is, for such a $\phi$, there is a formula $\Phi\left(x_{1}, x_{2}\right)$ of $S$ such that for all $m, n$,

(iii) $\phi(m)=n \Leftrightarrow \vdash{ }_{s} \Phi(\bar{m}, \bar{n})$;

and further,

(iv) $\vdash s\left(E_{1} x_{2}\right) \Phi\left(x_{1}, x_{2}\right)$.

This result not only yields that of Ehrenfeucht and Feferman as an immediate corollary but also gives a neat characterization of the class of partial recursive functions, in addition to showing that the condition (iii) of $[4$, p. 45] for definability of a total function by a formula $\Phi$ (viz. for each $n$

$$
\left.\vdash_{S} \Phi\left(\bar{n}, x_{2}\right) \wedge \Phi\left(\bar{n}, x_{3}\right) \supset x_{2}=x_{3}\right)
$$

implies the stronger condition obtained by replacing $\bar{n}$ by a variable

Presented to the Society, January 1, 1966; received by the editors June 29, 1966. 
$x_{1}$. The argument uses a theorem on exact separation of disjoint recursively enumerable sets due to Putnam and Smullyan; it is interesting to note that Shepherdson [3] obtains this separation result with his direct methods.

The present note gives a direct proof of a slight generalization of the theorem of Ritchie and Young alluded to above. Namely, let $S$ be any consistent recursively enumerable theory in which every recursive relation is definable (in the sense of Tarski-Mostowski-Robinson $\left[4\right.$, p. 44]) and which has a formula $x_{1} \leqq x_{2}$ satisfying (i) above as well as (ii)' below:

(ii) $\vdash_{s} x_{1} \leqq x_{2} \bigvee x_{2} \leqq x_{1}$

Alternatively, we may assume that $S$ satisfies just (i) and that every recursive function is definable in $S$ (cf. [3, p. 121]). In either case we have the following.

THEOREM. Every partial recursive function is strongly representable in $S$.

That is, if $\phi$ is a partial recursive function then there is a formula $\Phi\left(x_{1}, x_{2}\right)$ of $S$ such that for all $m, n$, (iii) above holds as well as (iv).

2. Weak representability. We say that the partial function $\phi$ is weakly represented in $S$ by $\Phi\left(x_{1}, x_{2}\right)$ provided that (iii) above holds as well as

$$
\text { (iva) } \vdash{ }_{s} \Phi\left(x_{1}, x_{2}\right) \wedge \Phi\left(x_{1}, x_{3}\right) \supset x_{2}=x_{3} \text {. }
$$

THEOREM 1. Every partial recursive function $\phi$ is weakly representable in $S$.

In what follows let $\phi$ be a fixed partial recursive function. Then according to the Enumeration Theorem of Kleene there is a recursive predicate $T(u, w)$ and a recursive function $U$ such that

$$
\phi(u)=v \Leftrightarrow(\exists w)[T(u, w) \& U(w)=v] .
$$

(We may take $T(u, w)$ as the $T_{1}(e, u, w)$ of [IM, p. 330], and the $U$ found there, where $e$ is any index for $\phi$.) We may assume that in particular $T$ has the property that $T\left(u, w_{1}\right) \& T\left(u, w_{2}\right) \Rightarrow w_{1}=w_{2}$. Let the recursive relations $T(u, w)$ and $U(w)=v$ be defined in $S$ by $\Im\left(x_{1}, x_{3}\right)$ and $\mathcal{U}\left(x_{3}, x_{2}\right)$, respectively, and let $D(x, y, z)$ be the conjunction of the two formulas

$$
J(x, z) \wedge\left(z_{1}\right)\left[z_{1} \leqq z \supset\left[\Im\left(x, z_{1}\right) \supset z_{1}=z\right]\right]
$$

and

$$
u(z, y) \wedge\left(y_{1}\right)\left[y_{1} \leqq y \supset\left[u\left(z, y_{1}\right) \supset y_{1}=y\right]\right] .
$$


Lemma 1. For any $u, v, w$,

$$
T(u, w) \& U(w)=v \Leftrightarrow \vdash_{s} D(\bar{u}, \bar{v}, \bar{w}) .
$$

Proof. It suffices to show the implication to the right, so suppose that $T(u, w)$ and $U(w)=v$. We have then $\vdash_{S} \mathfrak{J}(\bar{u}, \bar{w}) \wedge \mathcal{U}(\bar{w}, \bar{v})$ by the choice of $\Im$ and $\mathcal{~}$. But also for every $n \leqq w$ we have

$$
\vdash_{S}[\Im(\bar{u}, \bar{n}) \supset \bar{n}=\bar{w}]
$$

(since the propositional components of this, for each fixed $n$, can be proved or disproved appropriately), so by (i) we have

$$
\vdash_{S}\left(z_{1}\right)\left[z_{1} \leqq \bar{w} \supset\left[\Im\left(\bar{u}, z_{1}\right) \supset z_{1}=\bar{w}\right]\right] .
$$

In the same way we have

$$
\vdash_{s}\left(y_{1}\right)\left[y_{1} \leqq \bar{v} \supset\left[\mathcal{u}\left(\bar{w}, y_{1}\right) \supset y_{1}=\bar{v}\right]\right]
$$

and thus $\vdash_{S} D(\bar{u}, \bar{v}, \bar{v})$.

LEMmA 2. $\vdash_{S} D\left(x, y_{2}, z_{2}\right) \wedge D\left(x, y_{3}, z_{3}\right) \supset y_{2}=y_{3}$.

Proof. From (ii)' we have $\vdash{ }_{s} z_{2} \leqq z_{3} \bigvee z_{3} \leqq z_{2}$. Thus by specializing the variable $z_{1}$ in $\mathscr{D}\left(x, y_{2}, z_{2}\right)$ to $z_{3}$, and in $\mathscr{D}\left(x, y_{3}, z_{3}\right)$ to $z_{2}$, we obtain $\vdash_{S} D\left(x, y_{2}, z_{2}\right) \wedge D\left(x, y_{3}, z_{3}\right) \supset z_{2}=z_{3}$. From this equality and by a similar manipulation of the final clauses of $\mathscr{D}\left(x, y_{2}, z_{2}\right)$ and $D\left(x, y_{3}, z_{3}\right)$ we obtain the desired result.

Proof of Theorem 1 . Let $Q, P$ be recursive predicates defined as follows:

$Q(u, v, w, q) \Leftrightarrow q$ is (the Gödel number of) a proof (in $S$ ) of $D(\bar{u}, \bar{v}, \bar{w}$ ) $P(u, v, r, p) \Leftrightarrow p$ is (the Gödel number of) a proof (in $S$ ) of $A_{r}^{(3)}(\bar{u}, \bar{v}, \bar{r})$ where $A_{r}^{(3)}\left(x_{1}, x_{2}, x_{3}\right)$ is the formula of $S$ whose Gödel number is $r$ and which contains no variables free other than $x_{1}, x_{2}, x_{3}$. Then $P, Q$ are represented in $S$ by some $\odot, Q$, respectively. Let $r_{0}$ be the Gödel number of the formula

$$
\begin{aligned}
(E z) D\left(x_{1}, x_{2}, z\right) & \wedge\left(x_{4}\right)\left[\odot\left(x_{1}, x_{2}, x_{3}, x_{4}\right)\right. \\
& \left.\supset\left(E x_{5}\right)\left[x_{5} \leqq x_{4} \wedge\left(E x_{6}\right)\left[x_{6} \leqq x_{6} \wedge Q\left(x_{1}, x_{2}, x_{6}, x_{6}\right)\right]\right]\right] .
\end{aligned}
$$

We claim that $\phi$ is weakly represented by $\Phi$, where $\Phi\left(x_{1}, x_{2}\right)$ is the formula $A_{r_{0}}^{(3)}\left(x_{1}, x_{2}, \bar{r}_{0}\right)$.

Now suppose that $\vdash{ }_{s} \Phi(\bar{u}, \bar{v})$ for some $u, v$. Let $p$ be (the number of) a proof; then $\vdash_{s} \mathcal{P}\left(\bar{u}, \bar{v}, \bar{r}_{0}, \bar{p}\right)$ according to the definition of $P$ by $\odot$ and our supposition. Hence by specialization of $x_{4}$ to $\bar{p}$ in the definition of $\Phi$ we have (upon application of modus ponens) that 


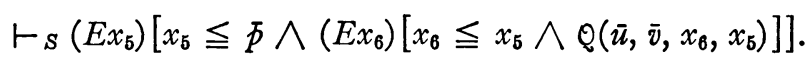

Hence (by a similar argument to the one above in connection with $D$ ), $Q(u, v, w, q)$ for some $w, q$ with $w \leqq q \leqq p$, since $Q$ is defined by $Q$. Hence (by the meaning of $Q$ ) there is a proof (in fact, with number $q$ ) of $D(\bar{u}, \bar{v}, \bar{w})$. We conclude both $T(u, w)$ and $U(w)=v$, and then that $\phi(u)=v$.

Conversely, let $\phi(u)=v$; take $w$ minimal so that $T(u, w)$ and $U(w)=v$. Then for some minimal $q \geqq w, q$ is a proof of $D(\bar{u}, \bar{v}, \bar{v})$ by Lemma 1 . Note in passing that $\vdash_{S}(E z) D(\bar{u}, \bar{v}, z)$ (by extension of the proof $q)$. Now for $p<q$, we have $\vdash_{S} \sim \mathcal{P}\left(\bar{u}, \bar{v}, \bar{r}_{0}, \bar{p}\right)$, since otherwise entails (as above) the existence of $w_{1}, q_{1}, p_{1}$ with $w_{1} \leqq q_{1} \leqq p_{1}<q$ where $q_{1}$ is a proof of $\mathfrak{D}\left(\bar{u}, \bar{v}, \bar{w}_{1}\right)$; but then $T\left(u, w_{1}\right)$ implies $w_{1}=w$, so $q \leqq q_{1}$ (contradicting the choice of $q$ ). Hence we can show by (i) and (ii) (or $\left.(\text { ii) })^{\prime}\right)$ that $\vdash_{S}\left(x_{4}\right)\left[\odot\left(\bar{u}, \bar{v}, \bar{r}_{0}, x_{4}\right) \supset \bar{q} \leqq x_{4}\right]$. But then

$$
\vdash_{S}\left(x_{4}\right)\left[\odot\left(\bar{u}, \bar{v}, \bar{r}_{0}, x_{4}\right) \supset \bar{q} \leqq x_{4} \wedge \bar{w} \leqq \bar{q} \wedge \mathcal{Q}(\bar{u}, \bar{v}, \bar{w}, \bar{q})\right],
$$

so we conclude (by existential quantifications), with the help of the previously noted fact that $\vdash_{s}(E z) D(\bar{u}, \bar{v}, z)$, that $\vdash{ }_{s} \Phi(\bar{u}, \bar{v})$.

All that remains to be shown is (iva), but this follows immediately from Lemma 2.

3. Strong representability. Now we show how to construct another Rosser-type argument to obtain the strong representability of $\phi$. To this end recall the definition of $A_{r}^{(3)}\left(x_{1}, x_{2}, x_{3}\right)$ given above. Define recursive $M, N$ as follows:

$M(u, v, r, p) \Leftrightarrow p$ is (the number of) a proof (in $S$ ) of $\sim \Phi(\bar{u}, \bar{v})$ $\supset A_{r}^{(3)}(\bar{u}, \bar{v}, \bar{r})$,

$N(u, v, r, q) \Leftrightarrow q$ is a proof of $\sim \Phi(\bar{u}, \bar{v}) \supset \sim A_{r}^{(3)}(\bar{u}, \bar{v}, \bar{r})$.

Let $M, N$ be defined in $S$ by $\mathscr{T}, \mathfrak{T}$, respectively, and let $r_{0}$ be the Gödel number of the formula

$$
\left(x_{4}\right)\left[\Re\left(x_{1}, x_{2}, x_{3}, x_{4}\right) \supset\left(E x_{5}\right)\left[x_{3} \leqq x_{2} \wedge \Re\left(x_{1}, x_{2}, x_{3}, x_{5}\right)\right]\right] .
$$

Take $R_{0}$ as $A_{r_{0}}^{(3)}\left(x_{1}, x_{2}, \bar{r}_{0}\right)$ and $\Phi^{*}\left(x_{1}, x_{2}\right)$ as the formula

$$
\begin{aligned}
\Phi\left(x_{1}, x_{2}\right) \vee\left[( z ) \sim \Phi ( x _ { 1 } , z ) \wedge \left[\left(x_{2}=\overline{0} \wedge\right.\right.\right. & \left.R_{0}\left(x_{1}, x_{2}\right)\right) \\
& \left.\left.\vee\left(x_{2}=\overline{1} \wedge \sim R_{0}\left(x_{1}, x_{2}\right)\right)\right]\right] .
\end{aligned}
$$

ThEOREM 2. $\Phi^{*}$ strongly represents $\phi$ in $S$.

Proof. By Theorem 1 we infer that if $\phi(u)=v$, then $\vdash{ }_{s} \Phi(\bar{u}, \bar{v})$, so also $\vdash{ }_{s} \Phi^{*}(\bar{u}, \bar{v})$. With the help of the same theorem $\vdash_{s} \Phi^{*}(x, y)$ $\wedge \Phi^{*}(x, z) \supset y=z$. It is straightforward to verify that $\vdash_{s}(E y) \Phi^{*}(x, y)$, merely using the logical form of $\Phi^{*}$, so it remains to be shown that if 
$\Phi^{*}(\bar{u}, \bar{v})$ is provable in $S$ for some $u, v$, then in fact $\phi(u)=v$. Thus suppose that $\vdash_{s} \Phi^{*}(\bar{u}, \bar{v})$; it suffices to show that $\vdash_{s} \Phi(\bar{u}, \bar{v})$ also. First note that if $v>1$, then $\vdash_{s} \bar{v} \neq \overline{0} \wedge \bar{v} \neq \overline{1}$ by our assumptions about $S$. But for such $v$ it easily follows from the form of $\Phi^{*}(\bar{u}, \bar{v})$ that $\vdash{ }_{S} \Phi(\bar{u}, \bar{v})$. Hence we need consider only the cases $v=0$ and $v=1$.

Case $v=0$. In this case we see that $\vdash_{s} \sim \Phi(\bar{u}, \overline{0}) \supset R_{0}(\bar{u}, \overline{0})$; let $p$ be the number of a proof. Note that $M\left(u, 0, r_{0}, p\right)$ holds then, so that $\vdash_{s} \operatorname{TC}\left(\bar{u}, \overline{0}, \bar{r}_{0}, \bar{p}\right)$ since $\Re$ defines $M$. Now extend the proof $p$ by specializing the $x_{4}$ in the definition of $R_{0}$ to $\bar{p}$, to obtain

$$
\vdash_{s} \sim \Phi(\bar{u}, \overline{0}) \supset\left(E x_{5}\right)\left[x_{5} \leqq p \wedge \Re\left(\bar{u}, \overline{0}, \bar{r}_{0}, x_{5}\right)\right] .
$$

Now two possibilities arise. First, that $\vdash s \sim \Phi(\bar{u}, \overline{0}) \supset \sim R_{0}(\bar{u}, \overline{0})$. In this subcase, clearly $\vdash{ }_{s} \Phi(\bar{u}, \overline{0})$ as desired. Otherwise, it is not the case that $\vdash_{s} \sim \Phi(\bar{u}, \overline{0}) \supset \sim R_{0}(\bar{u}, \overline{0})$, so $N\left(u, 0, r_{0}, q\right)$ is false for all $q$, and in particular for $q \leqq p$. Hence for such $q$, we have $\vdash_{s} \sim \mathcal{N}\left(\bar{u}, \overline{0}, \tilde{r}_{0}, \bar{q}\right)$, since $\Re$ defines $N$, and thus $\vdash_{s}\left(x_{5}\right)\left[x_{5} \leqq p \supset \sim \mathscr{T}\left(\bar{u}, \overline{0}, \bar{r}_{0}, x_{5}\right)\right]$. From this and $\left(0^{*}\right)$ we conclude that $\vdash_{S} \Phi(\bar{u}, \overline{0})$.

Case $v=1$. Now we see that $\vdash_{S} \sim \Phi(\bar{u}, \overline{1}) \supset \sim R_{0}(\bar{u}, \overline{1})$; if $q$ is the number of a proof, $N\left(u, 1, r_{0}, q\right)$ holds, so $\vdash{ }_{s} \Re\left(\bar{u}, \overline{1}, \bar{r}_{0}, \bar{q}\right)$. Again it is possible that $\vdash_{s} \sim \Phi(\bar{u}, \overline{1}) \supset R_{0}(\bar{u}, \overline{1})$; and, if so, then $\vdash_{S} \Phi(\bar{u}, \overline{1})$ as desired. Otherwise, there is no proof in $S$ of $\sim \Phi(\bar{u}, \overline{1}) \supset R_{0}(\bar{u}, \overline{1})$, and so $M\left(u, 1, r_{0}, p\right)$ fails for all $p$. In particular, for $p \leqq q$ we have $\vdash_{s} \sim \mathscr{M}\left(\bar{u}, \overline{1}, \bar{r}_{0}, \bar{p}\right)$, and so (by (i)) $\vdash{ }_{s} \operatorname{Tr}\left(\bar{u}, \overline{1}, \bar{r}_{0}, x_{4}\right) \supset \sim\left(x_{4} \leqq \bar{q}\right)$. Now from a proof of $\Re\left(\bar{u}, \overline{1}, \bar{r}_{0}, \bar{q}\right)$ we can construct one of $\bar{q} \leqq x_{4} \supset \bar{q}$ $\leqq x_{4} \wedge \Re\left(\bar{u}, \overline{1}, \bar{r}_{0}, \bar{q}\right)$, so $\vdash_{s} \bar{q} \leqq x_{4} \supset\left(E x_{5}\right)\left[x_{5} \leqq x_{4} \wedge \Re\left(\bar{u}, \overline{1}, \bar{r}_{0}, x_{5}\right)\right]$. By (ii) we have $\vdash_{S} x_{4} \leqq \bar{q} \bigvee \bar{q} \leqq x_{4}$; so by combining this with the above results,

$$
\vdash s \mathfrak{T}\left(\bar{u}, \overline{1}, \bar{r}_{0} x_{4}\right) \supset\left(E x_{5}\right)\left[x_{5} \leqq x_{4} \wedge \Re\left(\bar{u}, \overline{1}, \bar{r}_{0}, x_{5}\right)\right]
$$

Generalize on $x_{4}$ in $\left(1^{*}\right)$ to obtain $\vdash{ }_{S} R_{0}(\bar{u}, \overline{1})$, and conclude finally that $\vdash_{s} \Phi(\bar{u}, \overline{1})$ in this case also.

\section{Bibliography}

1. A. Ehrenfeucht and S. Feferman, Representability of recursively enumerable sets in formal theories, Arch. Math. Logik Grundlagenforsch. 5 (1960), 37-41.

2. R. W. Ritchie and P. R. Young, Strong representability of partial recursive functions, Notices Amer. Math. Soc. 13 (1966), 114.

3. J. C. Shepherdson, Representability of recursively enumerable sets in formal theories, Arch. Math. Logik Grundlagenforsch. 5 (1960), 119-127.

4. A. Tarski, A. Mostowski and R. M. Robinson, Undecidable theories, NorthHolland, Amsterdam, 1953.

IM. S. C. Kleene, Introduction to metamathematics, Van Nostrand, New York, 1952.

UNIVERSITY OF WASHINGTON 